\title{
INVOLUTIONS ON SPIN 4-MANIFOLDS
}

\author{
DANIEL RUBERMAN
}

(Communicated by Ronald Stern)

\begin{abstract}
We show that a simply-connected spin 4-manifold which admits a locally linear involution must have vanishing signature.

We also show that the codimensions of all components of the fixed point set of an involution on a spin 4-manifold are the same modulo 4 . There is no assumption of local linearity in this result, which extends a lemma of Atiyah and Bott.
\end{abstract}

This note records two results about involutions on spin 4-manifolds. The first result is an extension to the case of arbitrary involutions of a well-known lemma of Atiyah and Bott [AB]. Their lemma, proven in the smooth case in [AB], and extended to the locally linear case in [E2], states that the codimensions of all components of the fixed point set must agree modulo 4 .

The second result is a theorem which states that a simply-connected spin 4-manifold which admits a locally linear involution acting trivially on the homology must have vanishing signature. This was inspired by the fact that a holomorphic $\mathbf{Z}_{2}$-action on a $\mathrm{K} 3$ surface cannot be homologically trivial. Edmonds [E1] has constructed locally linear, homologically trivial actions of $\mathbf{Z}_{p}$ for $p$ an odd prime on virtually all simply connected 4-manifolds.

\section{CODIMENSIONS OF FIXED POINTS}

Theorem 1.1. Let $\tau: X \rightarrow X$ be an involution on the spin 4-manifold $X$ which preserves orientation and a spin structure on $X$. Then the codimensions of all components of the fixed point set of $\tau$ agree modulo 4 .

The outline of this Atiyah-Bott lemma in the smooth case is as follows: A spin structure $\sigma$ is a double covering $\hat{P} \rightarrow P$ of the principal frame bundle $P$ restricting to a non-trivial cover in each fiber; we say that $\tau$ preserves $\sigma$ if it lifts to a fiber preserving map $\hat{\tau}$ on this double cover. In each fiber $\hat{P}_{x}, \hat{\tau}$ has order 1 or 2 ; correspondingly, we say that $\tau$ has odd or even type. It is easy to see that the type must be the same in every fiber. Now, on the spheres linking the components of the fixed point set, $\tau$ is the antipodal map and hence has odd type if the codimension is 2 mod 4 and even type if the codimension is $0 \bmod 4$. Thus the result follows.

Received by the editors April 25, 1993.

1991 Mathematics Subject Classification. Primary 57S17, 57N13.

Author partially supported by an NSF Postdoctoral Research Fellowship. 
Essentially the same argument works in the topological case; one uses the topological tangent bundle and its corresponding principal frame bundle to define spin structures and type. (One also needs to know what codimension means in this context, as the fixed point set is not necessarily a manifold. However, Smith theory [B] says that the fixed point set must be a mod 2 homology manifold; since the action is orientation preserving, the dimension of a component must be 0 or 2.) What is missing is the linking sphere to calculate the type by looking in a neighborhood of the fixed point set. However, this is not really necessary: In a neighborhood of an isolated fixed point, one can easily find an invariant $3-$ manifold $M$. Since $M / \tau$ is spin, there is an invariant spin structure on $M$ with even type (cf. $[\mathrm{AB}, \S 8]$ ). However, this may not agree with the spin structure induced from $X^{4}$. For example, the spin structure on $S^{2} \times S^{1}$ invariant under the free involution $1_{S^{2}} \times-1_{S^{1}}$ has odd type. This involution could certainly be the restriction of a free involution on a spin 4-manifold. However, $S^{2} \times S^{1}$, with this action and the invariant spin structure, could not lie arbitrarily close to an isolated fixed point. To get around this difficulty, we apply the following theorem, due to the author (unpublished) and to S. Kwasik and R. Schultz [KS].

Theorem 1.2. Let $\tau$ be an orientation-preserving involution on a 4-manifold. Then in any neighborhood of an isolated fixed point, there is an invariant homology sphere $\Sigma$.

Proof. (Sketch) One classifies the 4-manifolds with the proper homotopy type of $\mathbf{R} P^{3} \times \mathbf{R}$ and observes that all of them are built with invariant homology spheres infinitely far out in the end.

The quotient $\Sigma / \tau$ is spin, so there is at least one $\tau$-invariant spin structure on $\Sigma$ of even type-the one which is pulled up from $\Sigma / \tau$. But a homology sphere has a unique spin structure, so we obtain:

Corollary 1.3. If $\tau$ is an involution on a spin 4-manifold and there is an isolated fuxed point, then $\tau$ has even type.

The argument for Theorem 1.1 is completed once we show that the existence of a codimension-two component of the fixed point set implies that $\tau$ has odd type. By Smith theory, such a component is a mod 2 homology manifold, so by Alexander duality with $Z_{2}$ coefficients, there is a circle in $X$ invariant under $\tau$ which is null-homologous in $X$. Since the circle is null-homologous, the spin structure induced on it must be the trivial one, so that $\tau$ is of odd type.

\section{LOCALLY LINEAR INVOLUTIONS ON SPIN MANIFOLDS}

It is a well-known consequence of the Lefschetz fixed-point theorem that a periodic map on a closed surface of genus greater than one must act non-trivially on the first homology. Likewise, holomorphic maps of finite order on a K3 surface never act trivially on the second homology; this may be deduced from the holomorphic Lefschetz fixed-point theorem [BPV]. It has been conjectured that a similar fact should hold for smooth actions of finite groups on a K3 surface. In this section we prove this conjecture for the case of involutions; in fact smoothness is inessential as we work with a locally linear involution throughout. We note that as a consequence of work of Edmonds [E1] this conjecture does not 
hold for odd-order locally linear group actions, so the smoothness hypothesis is necessary in general.

Theorem 2.1. Let $X$ be a spin 4-manifold with $H_{1}(X)=0$ which admits a locally linear involution $\tau$ such that $\tau_{*}$ is the identity on $H_{2}(X)$. Then the signature of $X$ is 0 .

Proof. First, we remark that the G-signature theorem holds for locally linear actions, with exactly the same statement as in the smooth case. For a homologically trivial action such as $\tau$, the G-signature $\sigma(\tau, X)=\sigma(X)$. By the locally linear version of Theorem 1.1, the fixed points of $\tau$ consist of a collection of isolated points or of a collection of 2-manifolds. Now 0-dimensional components of $\operatorname{fix}(\tau)$ contribute 0 to the G-signature of an involution, so the signature must vanish if there are only isolated fixed points.

The case of codimension-two fixed points is a little more complicated, and we rely on some results of Edmonds [E2]. By analyzing the spectral sequence of the Borel fibering associated to the $Z_{2}$ action on $X$, he shows the following:

(1) $\operatorname{Fix}(\tau)$ consists of 2 -spheres $\left\{S_{i}^{2}\right\}$. The number (say, $k$ ) of them is equal to $\frac{1}{2}$ the Euler characteristic of $X$.

(2) Any set of $k-1$ of the 2-spheres is linearly independent over $\mathbf{Z}_{2}$.

Since the 2-spheres are all disjoint and the intersection form of $X$ is even, the mod 2 intersection form on the subspace of $H_{2}\left(X ; \mathbf{Z}_{2}\right)$ spanned by fix $(\tau)$ must vanish. But this form is non-degenerate, by Poincare duality, so there can be at most $\frac{1}{2} \beta_{2}=\frac{1}{2} \chi(X)-1$ independent classes. Thus, in mod 2 homology, $\sum_{i} S_{i}^{2}=0$. It is a straightforward consequence of Alexander duality and item (2) above that there is a 2 -fold branched covering $\widetilde{X} \rightarrow X$, branched along all of the components of fix $(\tau)$.

From the multiplicativity of the Euler characteristic for unbranched covers and the fact that $2 k=\chi(X)$, we obtain $\chi(\widetilde{X})=\chi(X)$. On the other hand, it is a consequence of Smith-theory type arguments (cf. [G]) that $H_{1}\left(\widetilde{X} ; \mathbf{Z}_{2}\right)=$ 0 , so $H_{1}(\widetilde{X} ; \mathbf{Q})=0$. Thus the second homology groups of $X$ and $\widetilde{X}$ are isomorphic, and so, by standard transfer arguments it follows that the covering involution $\tilde{\tau}: \widetilde{X} \rightarrow \widetilde{X}$ acts trivially on $H_{2}(\widetilde{X} ; \mathbf{Q})$. It also follows that $\widetilde{X}$ and $X$ have the same signature. But we can compute the signatures a different way, by applying the G-signature formula to the involutions $\tau$ and $\tilde{\tau}$. Note that for a component $S_{i}^{2}$ of fix $(\tau)$ and its preimage $\widetilde{S}_{i}^{2}$, we have that $e\left(\widetilde{S}_{i}^{2}\right)=\frac{1}{2} e\left(S_{i}^{2}\right)$, since the branching index is 2 for all components of fix $(\tau)$. Since both $\tau$ and $\tilde{\tau}$ are homologically trivial, we obtain

$$
\sigma(\tilde{X})=\sigma(\tilde{\tau}, \widetilde{X})=\sum_{i} e\left(\widetilde{S}_{i}^{2}\right)=\frac{1}{2} \sum_{i} e\left(S_{i}^{2}\right)=\frac{1}{2} \sigma(\tau, X)=\frac{1}{2} \sigma(X) .
$$

But since $\sigma(\tilde{X})=\sigma(X)$, we must have that $\sigma(X)=0$.

\section{ACKNOWLEDGMENT}

I would like to thank Allan Edmonds for many interesting conversations on the general subject, which stimulated these results. I have recently received a preprint from T. Matumoto which shows that the condition in Theorem 2.1 on the first homology may be relaxed to the vanishing of mod 2 homology. 


\section{REFERENCES}

[AB] M. Atiyah and R. Bott, A Lefschetz fixed point formula for elliptic complexes: II, Ann. of Math. (2) 88 (1968), 451-491.

[BPV] W. Barth, C. Peters, and A. Van de Ven, Compact complex surfaces, Springer-Verlag, New York and Berlin, 1984.

[B] G. Bredon, Introduction to compact transformation groups, Academic Press, New York, 1972.

[E1] A. Edmonds, Construction of group actions on four-manifolds, Trans. Amer. Math. Soc. 299 (1987), 155-170.

[E2] —, Aspects of group actions on four-manifolds, Topology Appl. 31 (1989), 109-124.

[G] P. Gilmer, Configurations of surfaces in 4-manifolds, Trans. Amer. Math. Soc. 264 (1981), 353-380.

[KS] S. Kwasik and R. Schultz, Desuspension of group actions and the ribbon theorem, Topology 27 (1988), 443-457.

Department of Mathematics, Brandeis University, Waltham, Massachusetts 02254

E-mail address: ruberman@math.brandeis .edu 